\title{
Effect of a wake on drag and deformation of liquid column at high Weber numbers
}

\author{
Tomohiro KAMIYA*, Makoto ASAHARA** and Takeshi MIYASAKA** \\ *Mechanical and Civil Engineering Division, Gifu University \\ 1-1 Yanagido, Gifu-shi, Gifu 501-1112, Japan \\ E-mail:w3912006@edu.gifu-u.ac.jp \\ **Department of Mechanical Engineering, Gifu University \\ 1-1 Yanagido, Gifu-shi, Gifu 501-1112, Japan
}

Received: 11 September 2019; Revised: 30 October 2019; Accepted: 21 January 2020

\begin{abstract}
The objective of this study is to clarify the effect of a wake on liquid column drag and deformation for a highWeber-number ( $W e$ ) flow. A simulation was performed for a liquid column with a high $W e$ behind a shock wave, using a ghost fluid method as a two-phase flow solver. The simulated We were 500, 1000, 2000, 3000, and 4000 . Relatively large oscillation of the drag coefficients was observed for $W e=500$ and 1000 . This feature of the drag coefficients was possibly caused by varying pressure on the downstream interface. The pressure variation is derived from the wake. In addition, it was suggested that such varying pressures could contribute to the flattening of the liquid column.
\end{abstract}

Keywords: Liquid column deformation, High Weber number, Wake, Spray combustion

\section{Introduction}

In supersonic combustion ramjet (scramjet) engines, liquid hydrocarbon fuel is injected perpendicular to a high-speed gas flow (Osaka et al., 2007; Imamura et al., 2010). When this liquid jet of fuel is in a crossing flow, a fine mist is formed via two processes. First, liquid fragments are generated by the disintegration of the injected liquid jet (primary breakup); subsequently, these liquid fragments are dispersed into fine droplets through interactions with ambient gas (secondary breakup) (Broumand and Birouk, 2016). The particle diameters and concentrations formed via the secondary breakup process affect the evaporation rate and combustion behavior of the fuel. Therefore, many studies have been conducted concerning this secondary breakup process. Especially for the high-Weber-number $(W e)$ flow observed in the scramjet engine, studies of this liquid drop breakup have been conducted as follows: reclassification of liquid drop deformation and breakup regimes (Theofanous et al., 2004, 2008, 2012); investigations of the effect of Rayleigh-Taylor instability on liquid drop deformation (Joseph et al., 1999; Theofanous et al., 2004, 2008, 2012); quantification of liquid drop deformation (Hising and Faeth, 1995); and evaluations of the deforming liquid drop drag and acceleration (Hising and Faeth, 1995; Quan and Schmidt, 2006; Shao et al., 2017; Wadhwa et al., 2007; Chen, 2008; Meng and Colonius, 2015, 2018; Liu et al., 2018). This study focuses on the drag coefficients in high-We flows. In actual combustion engines, fine droplets are generated via the breakup of moving fragments. Therefore, a drag model of liquid drops in gas flow is used to track the moving liquid fragments. However, liquid drop drag in high-We flows has not been studied thoroughly. Additionally, this study examines the effect of the wake on the liquid column deformation.

Although the drag coefficient of a solid sphere in gas flow is relatively simple and has been widely studied for a long time, it cannot be directly applied as the drag coefficient of a liquid drop in gas flow because of the deformation and disintegration of liquid drops and the internal flow effect. Hence, the drag coefficients of deforming liquid drops in gas flow have been measured. Hsiang and Faeth (1995) observed liquid drop deformation and breakup for shock waveinitiated disturbances in air. They obtained the drag coefficient from the trajectory of the center of mass for a liquid drop at a Reynolds number of 1000-2000. For this range, the increasing effect of the drag coefficient derived from deformation 
is larger than the decreasing effect of the drag coefficient derived from internal flow. Three-dimensional simulations of liquid drops rapidly accelerated by a gas flow were conducted by Quan and Schmidt (2006) and Shao et al. (2017). The liquid drop drag coefficients were measured from the trajectory of the drop. Quan and Schmidt (2006) claimed that a recirculation, generated at the rear of a liquid drop, contributed to the increase in its drag coefficient. Shao et al. (2017) investigated the effect of $W e$, the density ratio of gas to liquid, and the ratio of gas to liquid viscosity on an unsteady drag coefficient. They clarified that the density ratio had the largest effect on the drag coefficient, and that We had the secondlargest effect on drag coefficient. Additionally, for a low-viscosity liquid (Ohnesorge number $<0.1$ ), the effect of liquid viscosity on drag coefficients is small. Wadhwa et al. (2007) simulated a transient deformation drop, which was accelerated in stationary gas at initialization for various We and Ohnesorge numbers. As We increases, the drag and deformation of a liquid drop increases. Additionally, increasing liquid viscosity leads to decreasing drag and a lower deformation rate because the viscous dissipation becomes larger in the liquid phase. However, We values of these studies are one hundred or less; these conditions differ significantly from the high-We flow.

Joseph et al. (1999) visualized liquid drop breakup at high We, and they derived a liquid drop acceleration by performing a polynomial approximation of the trajectory of the liquid drop. However, unsteady features of the acceleration of the liquid drop caused by the liquid drop deformation and the unsteady flow field were not considered. Chen (2008) as well as Meng and Colonius (2015) simulated liquid column deformation and breakup in a high-speed gas flow, and they derived an unsteady drag coefficient of the liquid column. Chen (2008) showed that the liquid column drag coefficient in gas flow oscillates as a function of time, similar to the drag coefficient of a solid column. When deformation by gas flow of the liquid column begins, a difference of drag coefficients between the liquid and solid columns occurs. However, the drag coefficient derived by Chen (2008) is limited to the initial phase of liquid column deformation. Meng and Colonius (2015) additionally provided a liquid column drag coefficient that included the time when the liquid column was sufficiently deformed.

Liu et al. (2018) simulated liquid drop breakup in a high-speed and high-dynamic-pressure flow, and they derived the acceleration of drops that are deformed and disintegrated in gas flow. The acceleration became high immediately when the liquid drop was exposed to a high-speed gas flow. Subsequently, although the acceleration was initially decreased, it then increased again. Liu et al. (2018) claimed that the subsequent increase in acceleration was derived from the extension of the liquid drop toward the crossflow direction, and the development of recirculation in the wake of the liquid drop. In addition, Meng and Colonius (2018) simulated shockwave interaction with a liquid drop and derived the acceleration of drops deformed and disintegrated in gas flow behind the shockwave. These simulations (Liu et al., 2018; Meng and Colonius, 2018) clarified that the acceleration of liquid drops in gas flow oscillates as a function of time. According to Liu et al. (2018) and Meng and Colonius (2018), this oscillation is caused by vortex shedding at the wake. As mentioned above, the liquid drop drag acceleration varies because drops are affected by the wake. However, the drag coefficient and the wake as a function of We have not been clarified.

Inamura et al. (2009) and Flock et al. (2012) observed a gas-flow velocity field around a liquid drop by using particle image velocimetry (PIV). Inamura et al. (2009) concluded that the stamen shape observed for a multimode breakup regime occurred for low We and was formed by high-pressure stagnation points owing to the strong backflow between the twin vortices at the wake. Flock et al. (2012) indicated that the wake structure may not significantly affect the transition between breakup regimes. To clarify the effect of the wake on the deformation and breakup of the drop, the force imparted on the drop by the wake must be considered, in addition to the structure of the wake. Thus, the gas-phase pressure on the interface must be quantified using numerical analysis.

In previous studies, axisymmetric flow simulations (Wadhwa et al., 2007; Han and Trygavason, 1999; Han and Trygavason, 2001) were conducted for low We using three-dimensional numerical analysis, with large computational costs. However, the axisymmetric hypothesis may be inappropriate for high We because the wake is non-axisymmetric. Igra and Takayama (2001) and Sembian et al. (2016) simulated a shockwave interaction with a liquid column. They considered shockwaves and expansion waves, which propagate the gas and liquid phase, rather than liquid deformation and breakup. As mentioned above, Chen (2008) and Meng and Colonius (2015) simulated liquid column deformation and breakup in high-speed gas flow. However, these simulations did not consider surface tension and viscosity, which resist liquid column deformation and breakup, and a compressible two-phase solver was used as the diffuse interface method. In diffuse interface methods, the interface location is unclear because the volume fractions representing the interface are diffused numerically. The derived drag coefficients bring quantitatively significant differences when the threshold of the volume fraction recognized as the interface varies (Meng and Colonius, 2015). 
In this study, a simulation is conducted for a liquid column with high We behind a shockwave by using the sharp interface method, which considers surface tension and viscosity. We examine the variation mechanism of the liquid column drag coefficients. Additionally, we clarify the effect of a wake on liquid column deformation by deriving the gas pressure at a downstream interface of the liquid column from the simulation results.

\section{Governing equations and numerical methods}

The two-dimensional Navier-Stokes equation is adopted as a governing equation:

$$
\begin{aligned}
& \frac{\partial \boldsymbol{Q}}{\partial t}+\frac{\partial \boldsymbol{E}}{\partial x}+\frac{\partial \boldsymbol{F}}{\partial x}=\frac{\partial \boldsymbol{E}_{v}}{\partial x}+\frac{\partial \boldsymbol{F}_{v}}{\partial x}, \\
& \boldsymbol{Q}=\left(\begin{array}{c}
\rho \\
\rho u \\
\rho v \\
E
\end{array}\right), \boldsymbol{E}=\left(\begin{array}{c}
\rho u \\
p+\rho u^{2} \\
\rho u v \\
(E+p) u
\end{array}\right), \boldsymbol{F}=\left(\begin{array}{c}
\rho v \\
\rho v u \\
p+\rho v^{2} \\
(E+p) v
\end{array}\right), \\
& \boldsymbol{E}_{v}=\left(\begin{array}{c}
0 \\
0 \\
\tau_{x x} \\
\tau_{x y} \\
\tau_{x x} u+\tau_{x x} v+\lambda T_{x}
\end{array}\right), \boldsymbol{F}_{v}=\left(\begin{array}{c}
\tau_{y y} \\
\tau_{y x} u+\tau_{y y} v+\lambda T_{y}
\end{array}\right),
\end{aligned}
$$

where $t$ denotes time, $\rho$ denotes density, $u$ denotes velocity in the $x$ direction, $v$ denotes velocity in the $y$ direction, $E$ denotes total energy, $p$ denotes pressure, $\tau$ denotes viscous stresses, $T$ denotes temperature, and $\lambda$ denotes thermal conductivity. The viscosity coefficients of gas and water are set to $1.8 \times 10^{-5}$ and $9.5 \times 10^{-4} \mathrm{~Pa} \cdot \mathrm{s}$, respectively, which are necessary to obtain the viscous stresses $\tau$. The thermal conductivity $\lambda$ is set as 0.026 and $0.60 \mathrm{~W} /(\mathrm{m} \cdot \mathrm{K})$ for gas and water, respectively. The viscosity coefficients and thermal conductivity are constant. In addition to Eq. (1), the stiffened gas equation for a state can be applied to the gas and liquid phase; the equation is given as:

$$
p=(\gamma-1) \rho e-\gamma \pi
$$

Here, $e$ denotes internal energy. The parameters are $(\gamma, \pi)=\left(2.35,10^{9}\right)$ for the liquid phase and $(\gamma, \pi)=(1.4,0)$ for the gas phase.

To ensure that the interface is tracked sharply, a ghost fluid method (Fedkiw et al., 1999) is used as the numerical method. Hence, the local force, which acts at the interface from the gas phase, can be obtained. This local force is beneficial for clarifying the mechanism of liquid column deformation. In this method, ghost fluids are set at the interface tracked by the interface tracking method, and the gas and liquid phases are solved separately. The ghost fluids are derived from the exact solutions of the Riemann problem at the interface, in accordance with Bo et al. (2011) and Terashima and Tryggvason (2010). In addition, an interface pressure jump is imposed on the Riemann problem to include surface tension (Bo et al., 2011):

$$
p_{g}-p_{l}=\sigma \kappa
$$

where $\sigma$ denotes surface tension, which is assumed constant and given as $0.07 \mathrm{~N} / \mathrm{m} ; \kappa$ denotes interface curvature; and the subscripts $g$ and $l$ denote the gas and liquid phases, respectively. A level-set method (Sussman et al., 1994) is used as the interface tracking method. In this method, an implicit distance function $\phi$ is introduced, where $\phi=0$ represents the interface, $\phi<0$ represents the liquid phase, and $\phi>0$ represents the gas phase. An interface advection is represented by: 


$$
\frac{\partial \phi}{\partial t}+\boldsymbol{u} \cdot \nabla \phi=0
$$

where $\boldsymbol{u}$ denotes a velocity vector. To maintain the properties of the distance function, the level-set function $\phi$ is reinitialized by iteratively calculating the partial differential equation (Sussman et al., 1994):

$$
\frac{\partial \phi}{\partial \tau}+S\left(\phi_{0}\right)(|\nabla \phi|-1)=0
$$

where $S\left(\phi_{0}\right)$ is a smooth sign function and $\phi_{0}$ is the level-set function at $\tau=0$. An interface curvature and a normal vector at the interface can be obtained easily in this method.

We solved the gas and liquid phase using the same methods. A Harten-Lax-van Leer contact Riemann solver (Toro et al., 1994) is utilized as the compressibility fluid solver. The highly accurate primitive values at the cell boundary are obtained by the third-order finite-difference monotonic upstream-centered scheme for the conservation law (Van Leer, 1977). The computational time step is derived from the Courant-Friedrichs-Lewy condition. The sound speed of the liquid phase is much higher than that of the gas phase, and hence, the time step of the simulation of this study is limited by the sound speed of the liquid phase.

The simulation sometimes fails owing to negative pressure near the interface. When this occurs, we reduce the time step size to continue the simulation.

\section{Validation problem}

\subsection{R22 bubble-shockwave interaction problem}

To validate the simulation code, the R22 bubble-shockwave interaction problem (Shyue, 2006) is considered. This problem involves the collision of a shockwave in air with a R22 gas bubble with a density higher than that of air; this condition is similar to the shockwave-liquid column interaction problem. The Euler equation is used as a governing equation for this problem as with the simulation of Shyue (2006). Figure 1 shows the computational setup. The shock wave, which propagates toward the left side, is initially located on the right side of the R22 bubble. An initial density, velocity of $x$ direction, and pressure at each domain is given as:

$$
(\rho, u, p)= \begin{cases}\left(1.225 \mathrm{~kg} / \mathrm{m}^{3}, 0 \mathrm{~m} / \mathrm{s}, 1.013 \times 10^{5} \mathrm{~Pa}\right) & \text { for pre-shocked air } \\ \left(1.686 \mathrm{~kg} / \mathrm{m}^{3},-113.5 \mathrm{~m} / \mathrm{s}, 1.59 \times 10^{5} \mathrm{~Pa}\right) & \text { for post-shocked air } \\ \left(3.863 \mathrm{~kg} / \mathrm{m}^{3}, 0 \mathrm{~m} / \mathrm{s}, 1.013 \times 10^{5} \mathrm{~Pa}\right) & \text { R22 bubble }\end{cases}
$$

The corresponding shockwave Mach number is 1.22. R22 is considered as an ideal gas, and the specific heat ratio $\gamma$ is 1.249 for the R22 bubble. Two computational grids are attempted: $3561 \times 357$ and $10681 \times 1069$, of which the number of cells per diameter are 200 and 600 .

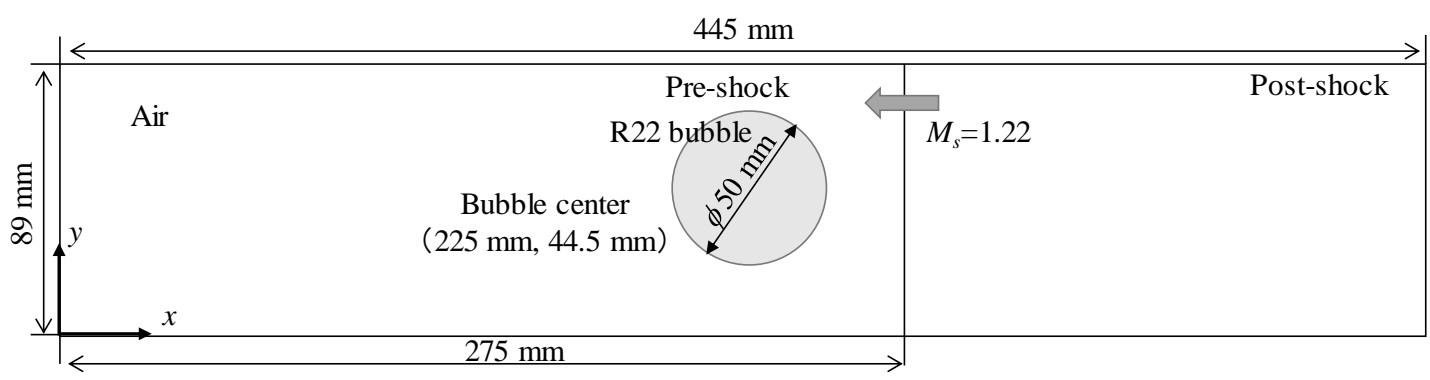

Fig. 1 Initial conditions of R22 bubble-shockwave interaction problem. 
Figure 2 shows that the interface shape and the density gradient distribution for the $10681 \times 1069$ grid at $t=130,250$, 420, and $1020 \mu \mathrm{s}$. When the images of Fig. 2 are compared with the numerical schlieren image of the simulation results of Shyue (2006), the interface shape and wave propagation behavior are qualitatively in agreement. As shown in Fig. 3, the distance from an upstream interface of an initial bubble to the upstream interface of a bubble is denoted by the upstream bubble wall length, and the distance from an upstream interface of an initial bubble to the downstream interface of a bubble is denoted by the downstream bubble wall length. Figure 3 shows the upstream and downstream bubble wall lengths, which are derived from the present simulation and the simulation of Shyue (2006). As shown in Fig. 3, the upstream and downstream bubble wall lengths, which are derived from the present simulation, are in good agreement with the lengths derived from the simulation of Shyue (2006).
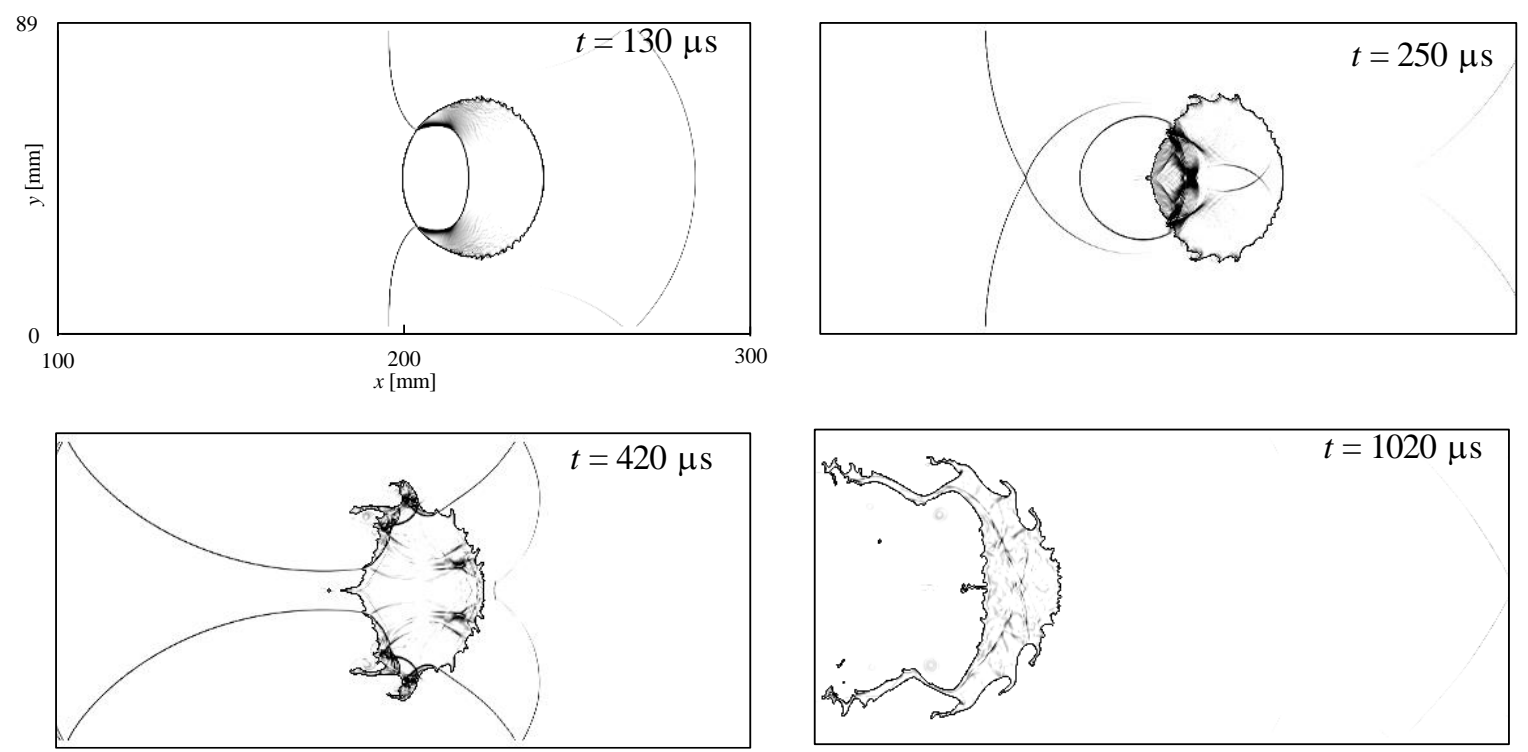

Fig. 2 Density gradient and interface shape of R22 bubble-shockwave interaction problem at $t=130,250,420$, and $1020 \mu$ s on a $10681 \times 1069$ grid.

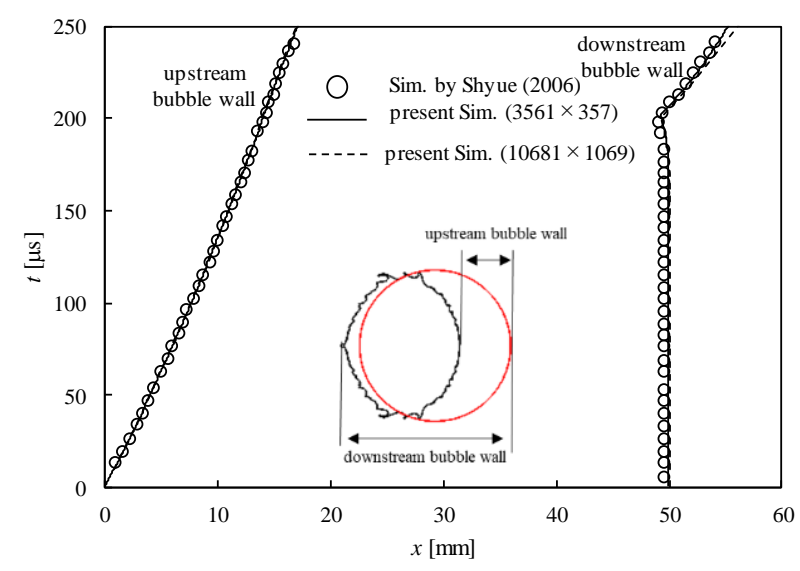

Fig. 3 Comparison with results of Shyue (2006) for a trajectory of the upstream and downstream bubble walls.

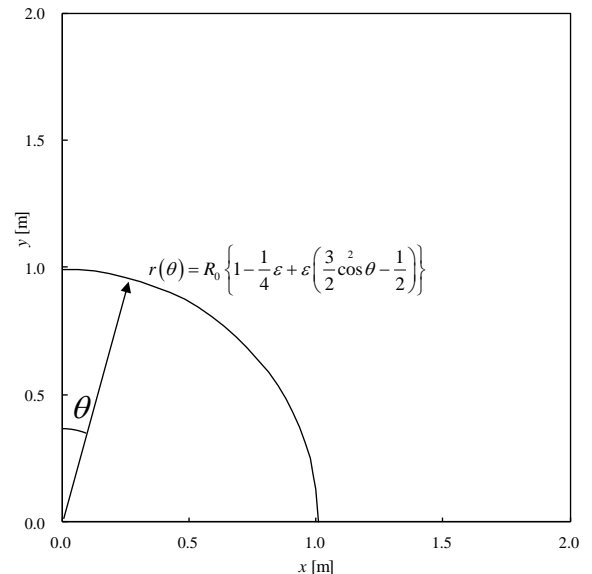

Fig. 4 Computational domain of liquid column oscillation problem.

\subsection{Liquid column oscillation problem}

To validate the numerical method for surface tension, the liquid column oscillation problem (Aulisa et al., 2006) is considered. In this problem, the initial interface shape is given as follows. 


$$
r(\theta)=R_{0}\left\{1-\frac{1}{4} \varepsilon+\varepsilon P_{l}(\cos \theta)\right\}=R_{0}\left\{1-\frac{1}{4} \varepsilon+\varepsilon P_{2}(\cos \theta)\right\}=R_{0}\left\{1-\frac{1}{4} \varepsilon+\varepsilon\left(\frac{3}{2} \cos ^{2} \theta-\frac{1}{2}\right)\right\}
$$

Here, $P_{l}(x)$ is a Legendre polynomial. The liquid column oscillates due to surface tension, and the oscillation amplitude is gradually decreased by viscous dissipation. Then, the oscillation period is derived:

$$
T=2 \pi \sqrt{\frac{\left(\rho_{\text {in }}+\rho_{\text {out }}\right) R_{0}^{3}}{\left(l^{3}-l\right) \sigma}}
$$

in theory. Here, the subscripts "in" and "out" denote the inside and outside of the liquid column. Figure 4 depicts the computational domain. This simulation is conducted over a quarter of the domain, because symmetric boundary conditions are imposed on the two symmetry lines. Two computational grids are attempted: $201 \times 201$ and $401 \times 401$. In this problem, $R_{0}=1 \mathrm{~m}, \varepsilon=0.01, \sigma=0.5 \mathrm{~N} / \mathrm{m}, \rho_{\text {in }}=\rho_{\text {out }}=1 \mathrm{~kg} / \mathrm{m}^{3}$, and $\mu_{\text {in }}=\mu_{\text {out }}=5 \times 10^{-3} \mathrm{~Pa} \cdot \mathrm{s}$ are given. Thus, the theoretical oscillation period is $5.13 \mathrm{~s}$. Figure 5 indicates the radial component of the interface location at $\theta=0$ as a function of time. From Fig. 5, the liquid column shape varies periodically. Additionally, the amplitude of this oscillation gradually decreases. Such behavior is qualitatively identical to the simulation results of Aulisa et al. (2006). The oscillation periods, which are derived from Fig. 5, are $5.67 \mathrm{~s}$ and $5.31 \mathrm{~s}$ for the $201 \times 201$ and $401 \times 401$ grids, respectively. The errors for the theoretical periods on each grid are $10.5 \%$ and $3.5 \%$, respectively. Thus, the error of the oscillation period decreases as the grid resolution improves. Additionally, the error of the oscillation period for the $401 \times 401$ grid is small. Hence, it is confirmed that the surface tension is appropriately solved.

We tried to simulate the liquid column oscillation problem for the condition of a large density ratio, as well as the simulation of Chang et al. (2013). Our simulation code cannot compute appropriately for large density ratio conditions. However, in this study, because liquid column flattening in the initial stage is the focus for high-We conditions, the effect of surface tension on liquid column flattening is relatively small. Hence, this numerical method may simulate liquid column flattening in the initial stage.

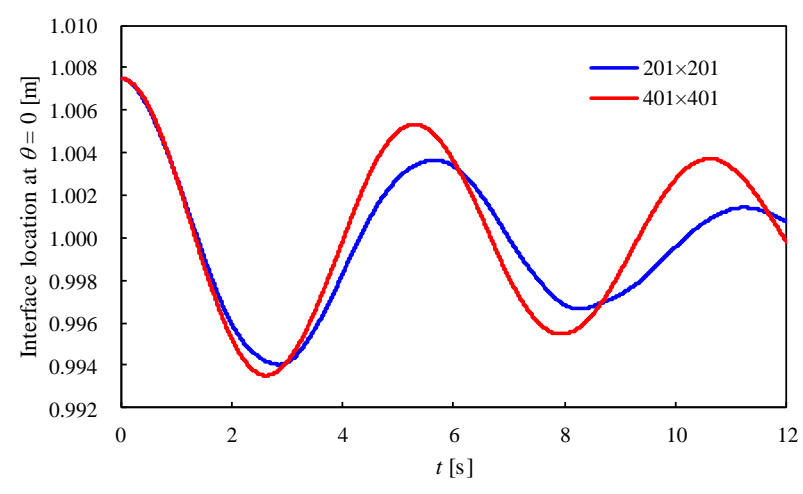

Fig. 5 Interface location at $\theta=0$ for liquid column oscillation problem.

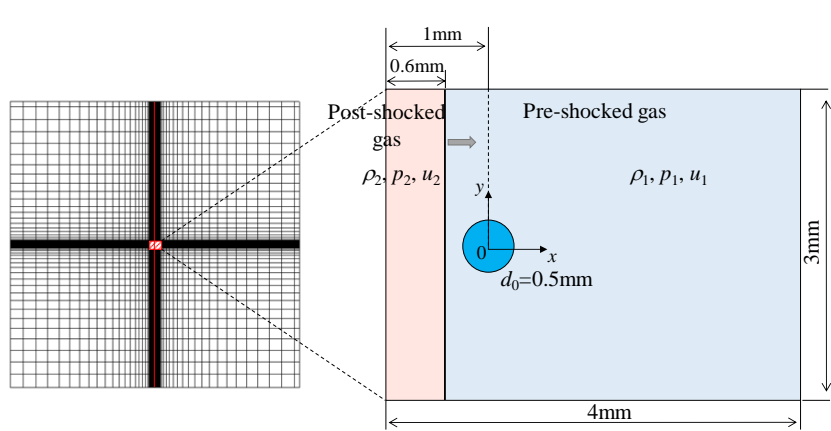

Fig. 6 Computational grid and initial condition for liquid column-shockwave interaction problem.

Table 1 Computational initial conditions of liquid column shockwave interaction problem.

\begin{tabular}{c|c|c|c|c}
\hline \hline Weber number & Reynolds number & $\rho_{2}\left[\mathrm{~kg} / \mathrm{m}^{3}\right]$ & $u_{2}[\mathrm{~m} / \mathrm{s}]$ & $p_{2}[\mathrm{MPa}]$ \\
\hline 500 & 10020 & 1.88 & 193 & 0.264 \\
1000 & 15109 & 2.13 & 256 & 0.320 \\
2000 & 23013 & 2.47 & 336 & 0.404 \\
3000 & 29548 & 2.72 & 393 & 0.472 \\
4000 & 35329 & 2.91 & 438 & 0.532 \\
\hline
\end{tabular}

\section{Computational setup}

We simulated an interaction between a liquid column and shockwave. Figure 6 shows the computational grid and the 
initial conditions. As shown in Fig. 6, a stretch grid is used to avoid boundary effects. To ensure that the dynamic pressures behind the shockwave are varied, the shockwave Mach numbers are varied by fixing the initial liquid column diameter $d_{0}$, density $\rho_{1}$, velocity $u_{1}$, and pressure $p_{1}$ in the pre-shocked gas region. We is defined as:

$$
W e=\frac{\rho_{2} u_{2}^{2} d_{0}}{\sigma}
$$

where $\rho_{2}$ and $u_{2}$ denote density and velocity behind the shockwave, respectively, as shown in Fig. 6 . Table 1 shows $W e$ and the Reynolds numbers, which are derived from the post-shockwave value and initial diameter, $\rho_{2}, u_{2}$, and pressure behind the shockwave $p_{2}$. In this study, we simulated five $W e$ conditions of 500, 1000, 2000, 3000, and 4000. Time was normalized by the characteristic time, which was first derived by Ranger and Nicholls (1968):

$$
t_{d}=\frac{d_{0}}{u_{2}} \sqrt{\frac{\rho_{l}}{\rho_{2}}} .
$$

Here, $\rho_{l}$ denotes liquid density. This characteristic time has been used for many previous studies.

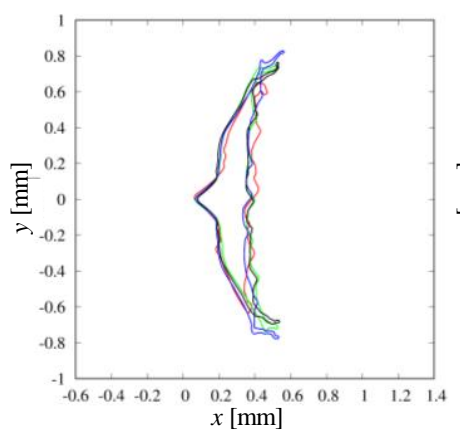

(a) $W e=1000$

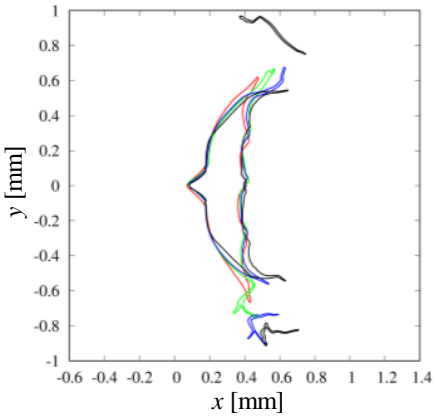

(b) $W e=2000$

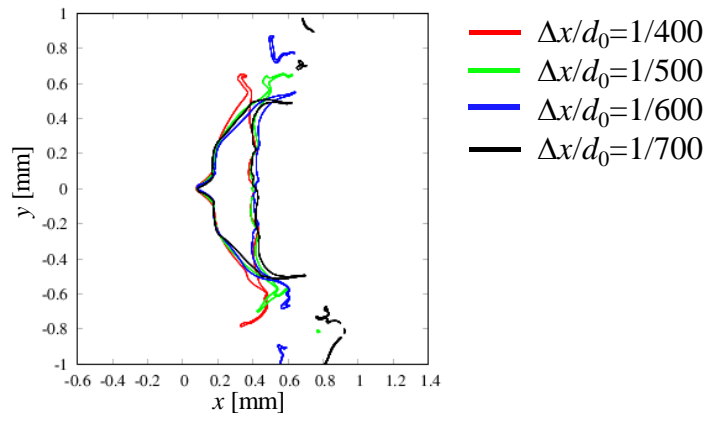

(c) $W e=3000$

Fig. 7 The liquid column-shockwave interaction problem results of interface shape for $W e=1000,2000$, and 3000 under various grid conditions.

\section{Grid convergence tests}

We attempt four grid resolutions for the same computational condition: $\Delta x / d_{0}=1 / 400,1 / 500,1 / 600$, and 1/700. Figure 7 shows the interface shapes for $W e=1000,2000$, and 3000 on each grid. The interface shapes for all grid conditions are similar, except for the tail shape at the lateral side of the liquid column. In addition, the trajectory of the center of mass for each grid is compared. To obtain the location of the center of mass, the core liquid column, which is the portion of the liquid column excluding the tail shape, is extracted. The core liquid column is defined as the portion surrounded by the line $y=y_{c} \pm b$ and the interface. The values of $b$ and $y_{c}$ are determined to satisfy the equations

$$
\begin{aligned}
& \int_{-b+y_{c}}^{b+y_{c}} \int_{x_{s}}^{x_{e}} \frac{1}{2}\left(1-\operatorname{sgn}\left(\phi^{\prime}\right)\right) d x d y=\psi S \\
& \int_{y_{s}}^{y_{c}} \int_{x_{s}}^{x_{e}} \frac{1}{2}\left(1-\operatorname{sgn}\left(\phi^{\prime}\right)\right) d x d y=0.5 S
\end{aligned}
$$

In the above equations, $x_{s}$ and $y_{s}$ are the minimum values of $x$ and $y$ in the computational region, respectively, and $x_{e}$ is the maximum value of $x$ in the computational region. $S$ denotes the total area of the liquid phase excluding the fragments formed by disintegration at the tail. $\phi$ ' denotes the level-set function with the sign modified to "+" for these liquid fragments. Figure 8 shows the core liquid column for $\psi=0.8$ as an example. The center of mass of the $x$ direction $x_{G, \psi}$ 
is derived following the integral:

$$
x_{G, \psi}=\int_{\text {core }} \frac{\rho x d S}{\rho d S} .
$$

Figure 9 shows the center of mass for $W e=2000$ on each grid for $\psi=0.8$. As shown in Fig. 9, the centers of mass for all grid conditions are almost identical. From the comparison of interface shapes and centers of mass when the grid resolutions were varied, the mesh size converges at $\Delta x / d_{0}=1 / 400$. In the following section, we adopt $\Delta x / d_{0}=1 / 500$, which represents grid convergence well.

In general, it is known that the level-set method does not preserve mass. Figure 10 shows the liquid mass normalized by the initial liquid mass as a function of dimensionless time. From Fig. 10, we confirmed that $98 \%$ of the liquid mass is preserved for $W e=2000$ on each grid.

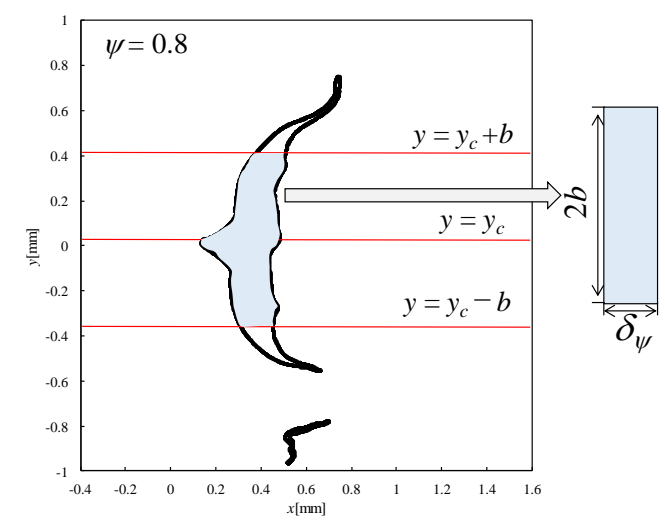

Fig. 8 Extraction of core liquid column.

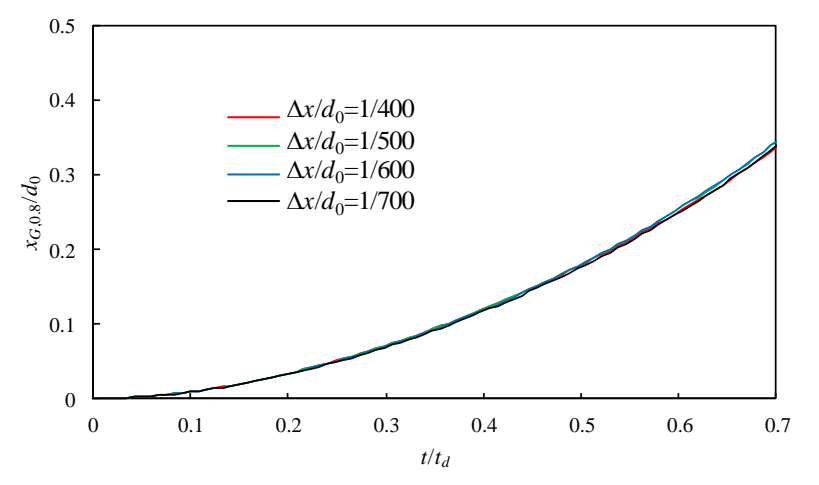

Fig. 9 Location of liquid column centers of mass of $W e=2000$ for various grid conditions.

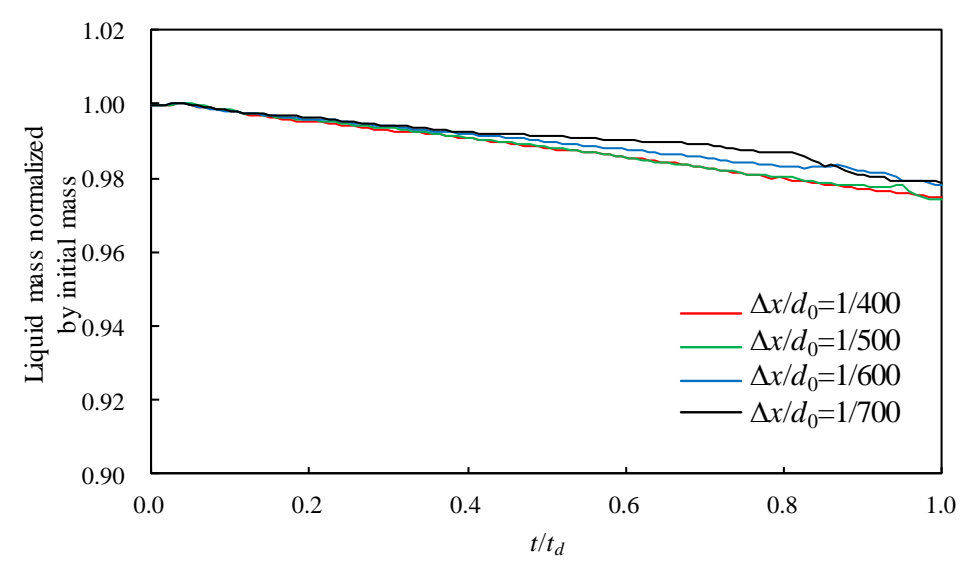

Fig. 10 Liquid mass normalized by initial liquid mass as a function of time.

\section{Results and Discussion}

\subsection{Qualitative features of liquid column deformation}

Figure 11 shows the interface shapes and non-dimensional pressure distributions:

$$
C_{p}=\frac{p-p_{2}}{p_{d}}
$$


for $W e=500,2000$, and 4000. From Fig. 11, the upstream pressure of the liquid column is shown to be higher than the pressure of the lateral side. At $t / t_{d}=0.4$, the liquid column was flattened. The interface of the upstream side and the interface of the lateral side of the liquid column formed a tip shape. The tips of the upstream interface were more notable as $W e$ was decreased. At $t / t_{d}=0.6$, the flattening of the liquid column progressed. In addition, the tip of the upstream interface became large. At $t / t_{d}=0.8$, the upstream interface formed a wavy shape, which was constructed as three tips for $W e=500$. Meanwhile, the upstream interfaces around the tips were smooth for $W e=2000$ and 4000 . At $t / t_{d}=1.0$, the corrugation of the upstream interface became large for $W e=500$. At $t / t_{d}=1.2$, the corrugation of the upstream interface dug into the liquid column for $W e=500$, and small corrugations were formed at the upstream interface around the tip for $W e=2000$ and 4000 . Additionally, from the interface shape at $t / t_{d}=1.4$, the corrugations of the upstream interface were larger than the corrugations at $t / t_{d}=1.2$. In past experiments examining drop breakup in a gas flow for high $W e$, corrugations of the upstream interface of the drop were observed (Park et al., 2006, Kamiya et al., 2019). Because the upstream interface of the liquid column is accelerated from the gas phase toward the liquid phase, Rayleigh-Taylor instability may be one of the causes of these corrugations. A relatively large tip is observed at the center of the upstream interface in the simulation results.

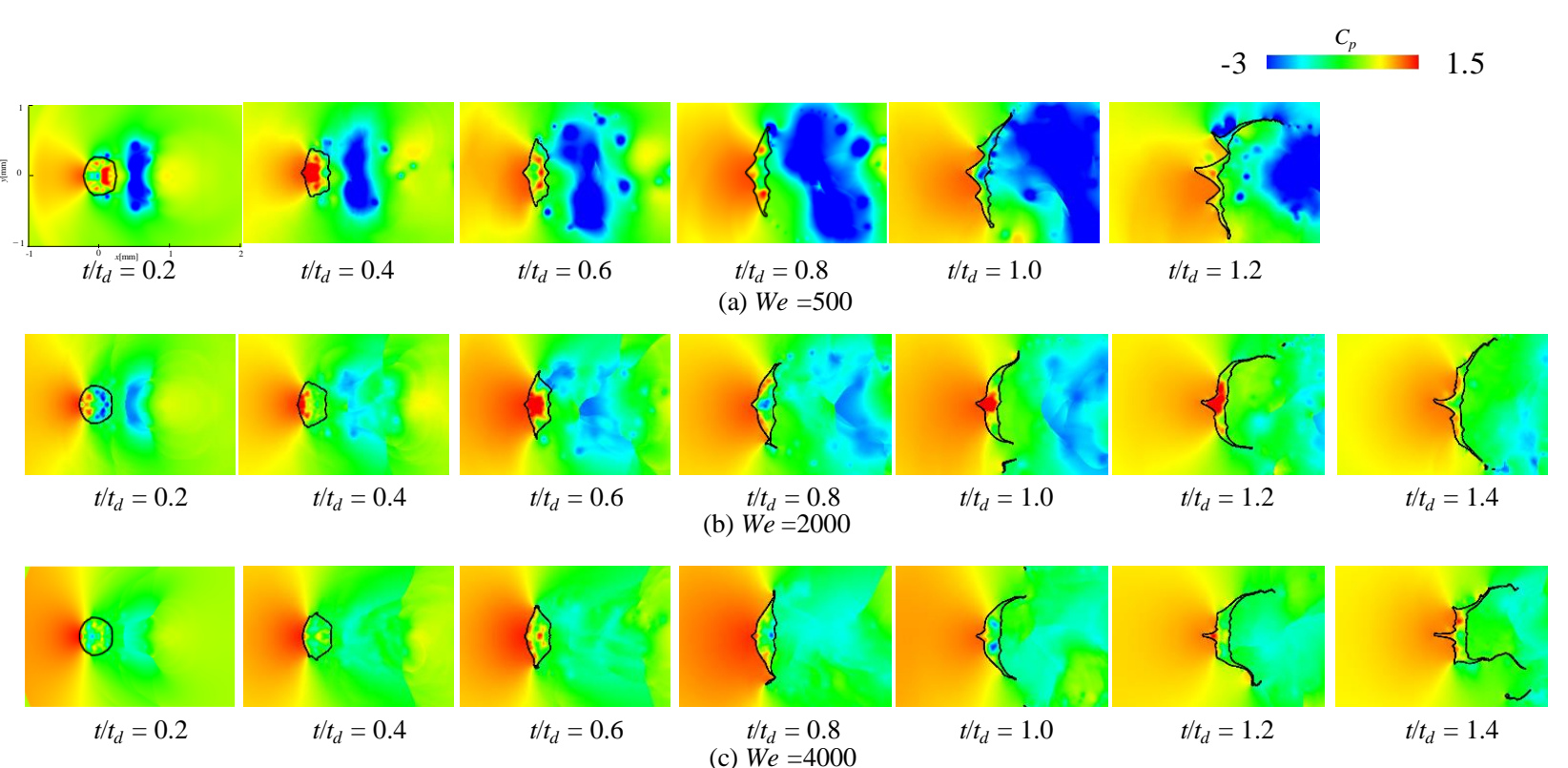

Fig. 11 Nondimensional pressure distributions and interface shapes for $W e=500,2000$, and 4000 .

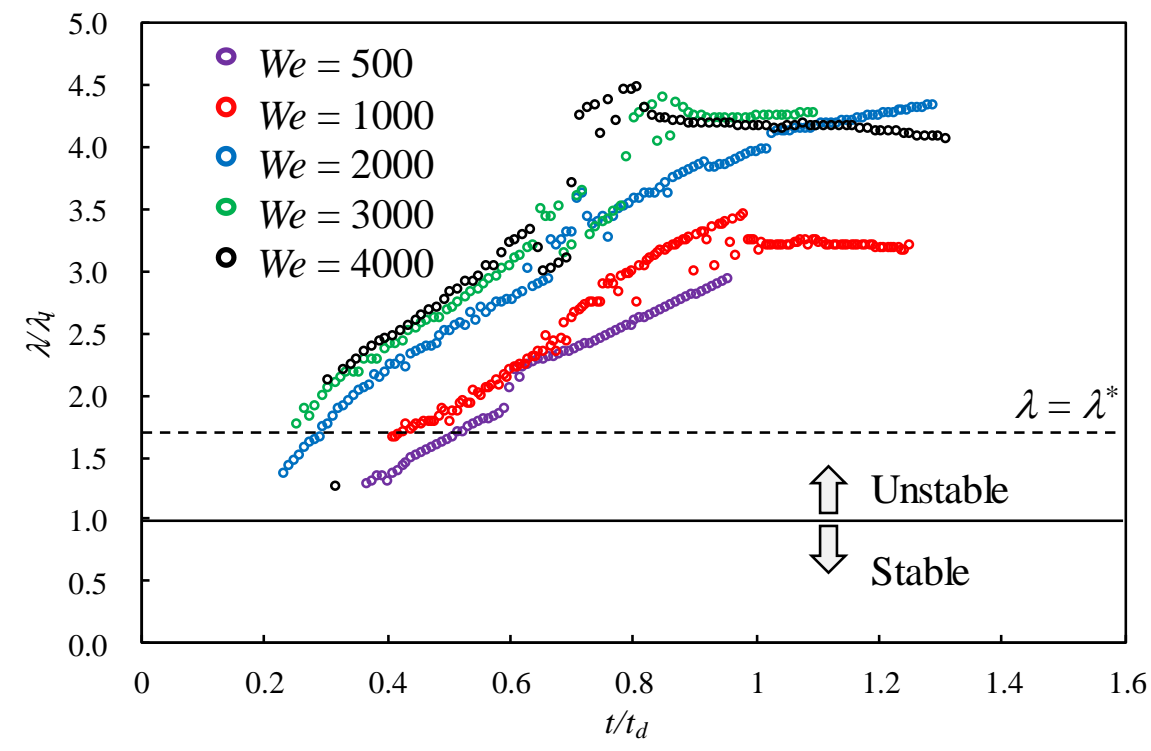

Fig. 12 Wavelength of the tip normalized by $\lambda_{l}$ as a function of time for various grid conditions. 
Using a linear theory of Rayleigh-Taylor instability, the applicability of the tip wavelength at the upstream interface is investigated. For an inviscid fluid, the growth factor of Rayleigh-Taylor instability:

$$
n=\sqrt{a k-k^{3} \frac{\sigma}{\rho_{l}}},
$$

is obtained from the linear theory by Chandrasekhar (1981). Here, $a$ and $k$ denote the acceleration and wavenumber of the disturbance. From this equation, the wavelength when the growth factor is maximum is:

$$
\lambda^{*}=2 \pi \sqrt{\frac{3 \sigma}{\rho_{l} a}} .
$$

When the growth factor is zero, the wavelength becomes:

$$
\lambda_{l}=2 \pi \sqrt{\frac{\sigma}{\rho_{l} a}} .
$$

If the wavelength is smaller than $\lambda_{l}$, no disturbance develops by Rayleigh-Taylor instability. Figure 12 shows the wavelength of the tip $\lambda$ normalized by $\lambda_{l}$. From Fig. 12, because $\lambda / \lambda_{l}>1$, the tip of the upstream interface can be developed by Rayleigh-Taylor instability. Additionally, $\lambda$ is of the same order as $\lambda^{*}$. Therefore, Rayleigh-Taylor instability is one of the possible causes of tip development. However, such a large tip at the center of the upstream interface has not been observed experimentally; therefore, we will conduct an axisymmetric and three-dimensional simulation to verify the simulation methods.

As mentioned above, we observed that the upstream interface of the liquid column became corrugated at high We. However, the corrugation pattern differed between $W e=500$ and the other conditions. Additionally, the upstream interface of the tip became large as We decreased when the time was normalized by the characteristic time in Eq. (10). Highaccuracy numerical simulation was also required to capture small-scale waves of the upstream interface.

\subsection{Drag coefficient and wake}

Liquid fragments are generated through liquid jet disintegration, and they subsequently break up into fine droplets in gas flow. Hence, the spatial distribution of fine droplets is influenced by the trajectories of the liquid fragments. To predict the trajectory of fragments, an unsteady drag coefficient model is required. A drag coefficient is derived from the acceleration of the core liquid column as:

$$
C_{D}=\frac{m_{\text {core }} a_{G, \psi}}{\frac{1}{2} \rho_{2}\left(u_{2}-u_{G, \psi}\right)^{2} d_{0}}
$$

where $m_{\text {core }}$ denotes the mass of the core liquid column discussed in Section 5, and $u_{G, \psi}$ denotes the velocity of the center of mass, defined as

$$
u_{G, \psi}=\int_{\text {core }} \frac{\rho u d S}{\rho d S} .
$$

The acceleration of the core liquid column $a_{G, \psi}$ is derived by the difference of the velocity of the center of the mass $u_{G, \psi}$, which is applied as a moving average. Here, $\psi$ is set to 0.8 . Meng and Colonius $(2015,2018)$ also derived drag coefficients from a liquid column and from drop acceleration. 
Figure 13 shows the drag coefficient for each $W e$. The relatively large drag coefficients oscillate for $W e=500$ and 1000 at $t / t_{d}=0-0.7$. Such oscillations were also observed for the acceleration and drag coefficients derived by the simulations of Chen (2008) and Meng and Colonius (2015). Additionally, Meng and Colonius (2015) mentioned that the amplitude of the oscillation became large as the Mach number of an incident shockwave decreases. In the simulation of Meng and Colonius (2015), the decreasing Mach number of the incident shockwave corresponded with decreasing dynamic pressure. Therefore, the drag coefficient of the present simulation demonstrates the same tendency as the previously simulated drag coefficient. Further, such oscillations of the drag coefficient are caused by vorticity shedding at the wake (Meng and Colonius, 2015). However, a relation between the wake and the drag was not investigated; the reason for the oscillation of the drag coefficient remains unclear.

The Strouhal number $(S t)$ was derived from the oscillation frequency of the drag coefficient obtained from Fig 13, the velocity behind the shockwave, and the initial liquid column diameter. Thus, St was 0.27 and 0.24 when $W e$ was 500 and 1000, respectively. St of the rigid column at the same Reynolds number is approximately 0.2 (Blevins, 1990). Hence, $S t$ obtained from the present simulation is close to that of a rigid column. Therefore, it is considered that the drag coefficient oscillates owing to the pressure of the liquid interface, which is periodically varied by vortices at the wake as well as the rigid column.

Figure 14 shows the color contour of such pressure at $t / t_{d}=0.17,0.22$, and 0.29 for $W e=1000$ and 2000 . The drag coefficient of $W e=1000$ becomes the local minimum value at $t / t_{d}=0.17$ and 0.29 , and it becomes the local maximum value at $t / t_{d}=0.22$. The nondimensional pressure of the downstream interface at $t / t_{d}=0.17$ and 0.29 , when the drag coefficient reaches a minimum value, is higher than that of $t / t_{d}=0.22$ for $W e=1000$. Therefore, the oscillation of the drag coefficient for $W e=1000$ is caused by varying pressure of the downstream interface.

To clarify the reason for the downstream pressure variation, the wake of the liquid column was investigated. Figure 15 shows the velocity magnitude color contour and the velocity vector for $W e=1000$; a pair of vortexes similar to a twin vortex was formed at the rear of the liquid column at $t / t_{d}=0.10$. A high-speed gas flow toward the liquid column was observed between the two vortexes at $t / t_{d}=0.17$. These vortexes were enlarged in the flow direction at $t / t_{d}=0.20$. The high-speed gas flow toward the liquid column was observed at the downstream edge of the twin vortexes at $t / t_{d}=0.22$. At $t / t_{d}=0.29$, the flattened vortexes returned to shapes approaching circular, and the high-speed gas flow toward the liquid column was observed again near the liquid column. Figure 15 shows the pressure of the downstream interface of the liquid column increasing for $W e=1000$ when the high-speed gas flow toward the liquid column was generated near the liquid column, corresponding to times $t / t_{d}=0.17$ and 0.29 . Therefore, the increment of pressure at the downstream interface may be derived by converting the kinetic energy of the gas flow to pressure energy because the high-speed gas flow is deaccelerated by the liquid column.

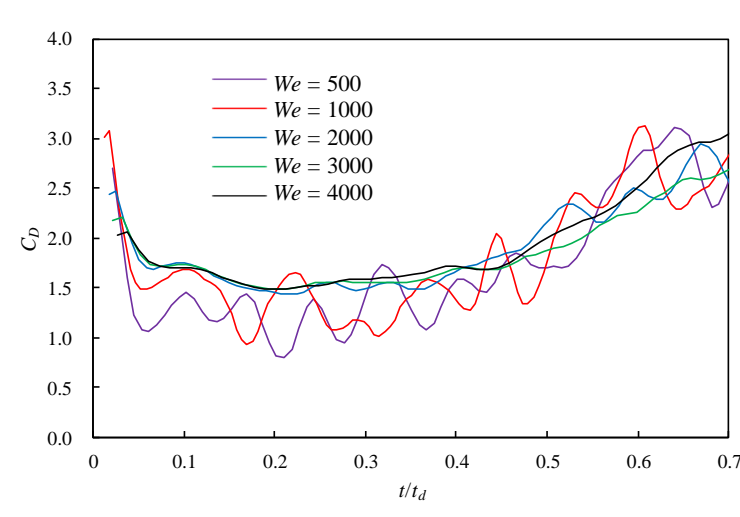

Fig. 13 Drag coefficient of transient deformation liquid column for various Weber number conditions.

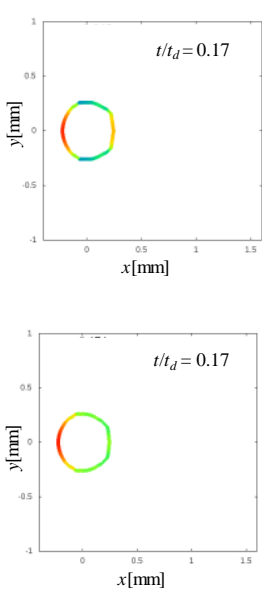

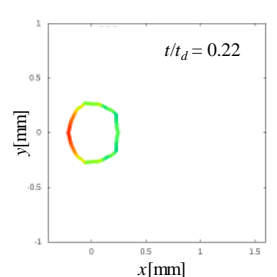

(a) $W e=1000$

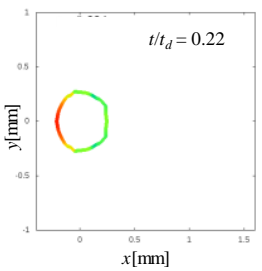

(b) $W e=2000$
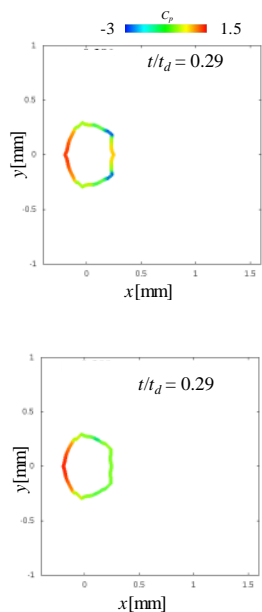

Fig. 14 Color contour of non-dimensional gas pressure on interface for $W e=1000$ and $W e=2000$. 

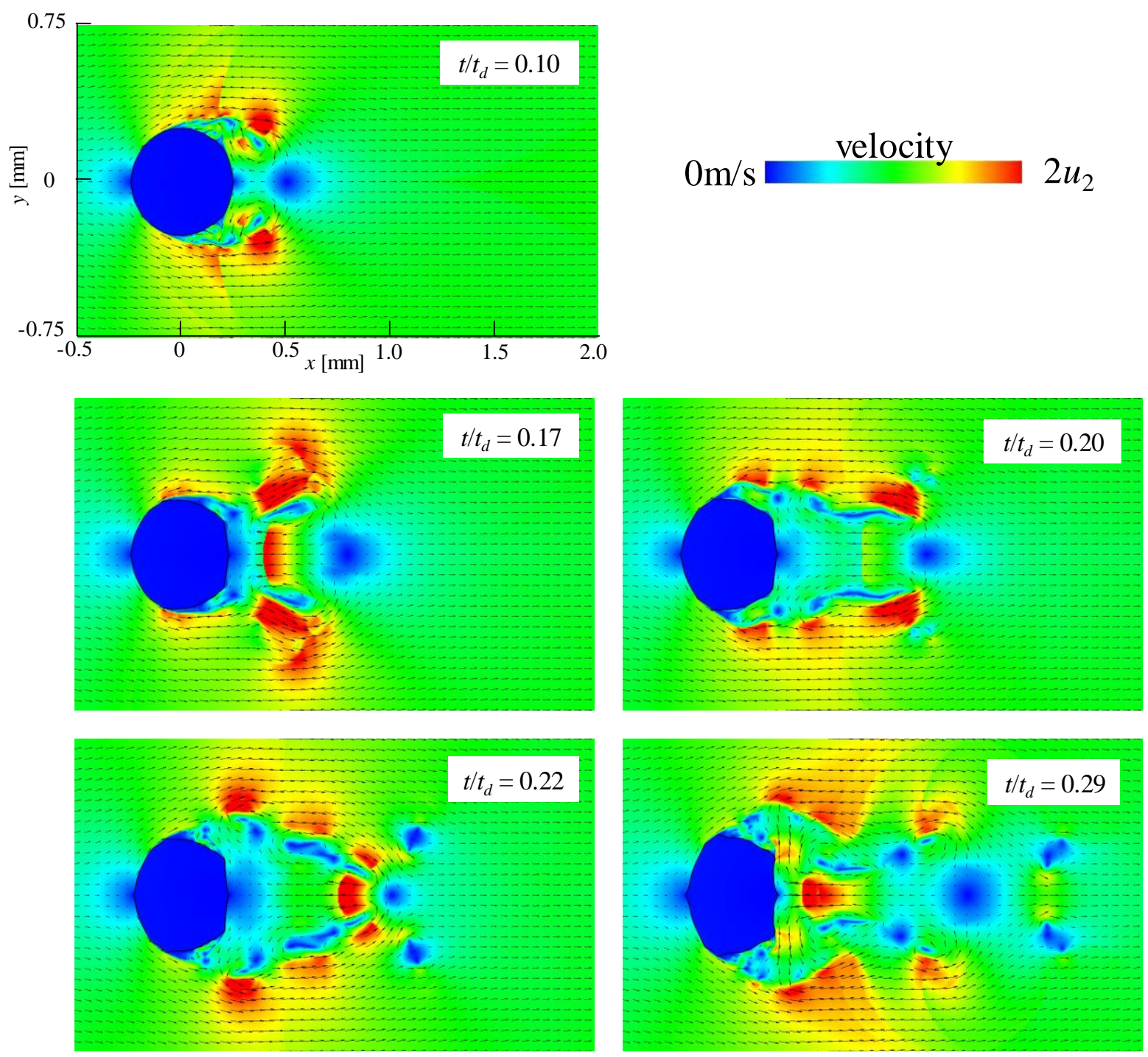

Fig. 15 Velocity magnitude color contour and velocity vector for $W e=1000$

$$
\text { at } t / t_{d}=0.10,0.17,0.20,0.22 \text {, and } 0.29 \text {. }
$$

\subsection{Trajectory and deformation of the liquid column}

As described in Section 6.2, the spatial distribution features of fine droplets are influenced by the trajectories of the liquid fragments. Additionally, the thickness and spanwise length were often measured in previous studies as a way of quantifying liquid droplet shape. In this section, the time history of the location of the center of mass, thickness, and spanwise length are derived. As shown in Fig. 8, the spanwise length is defined as the $y$ direction length $2 b$ and the thickness $\delta_{\psi}$, which is defined as:

$$
\delta_{\psi}=\frac{\psi S}{2 b}
$$

where $\psi=0.8$. Figure 16(a) shows the location of the center of mass, and Fig. 16(b) shows the spanwise length $2 b$ and the thickness $\delta$.8. As shown in Fig. 16(a), the liquid column moved downstream as time passed. Additionally, the location of the center of mass for all We conditions were similar when the time was normalized by the characteristic time, Eq. (10). Meanwhile, as shown in Fig. 16(b), the spanwise length $2 b$ increased and the thickness $\delta_{\psi}$ decreased as time passed, and the thickness $\delta_{\psi}$ tended to become small as We decreased. From Fig. 14, although the dimensionless pressure at the downstream interface periodically increased for $W e=1000$, such an increase was not observed for $W e=2000$. This 
increase in the downstream interface pressure is considered to promote liquid column flattening. Hence, the liquid column thickness as a function of dimensionless time for $W e=1000$ is smaller than that for other $W e$ conditions. This suggests that the pressure fluctuation at the downstream interface owing to the wake affects the liquid column deformation.

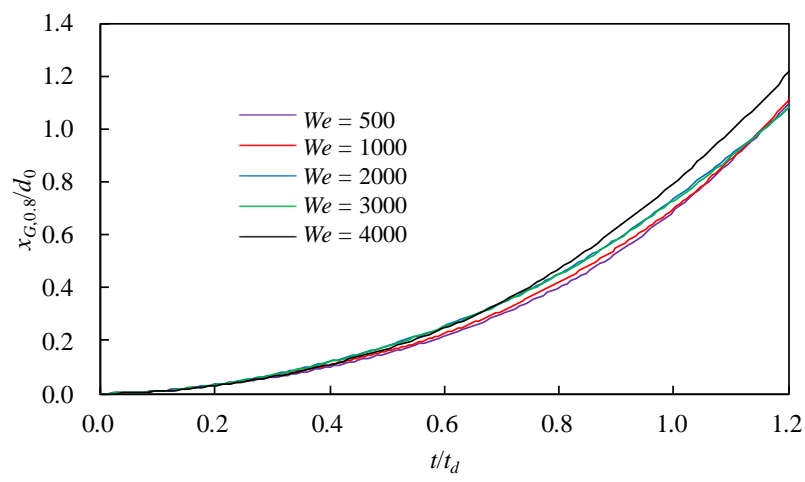

(a) Liquid column center of mass

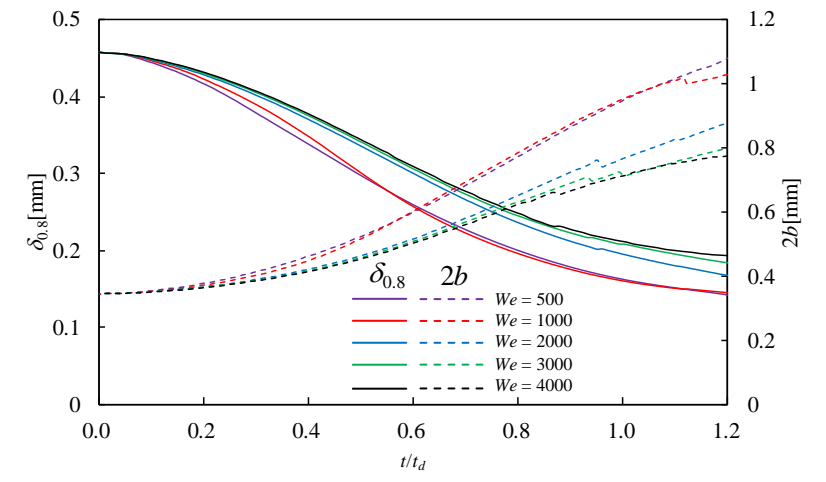

(b) Thickness and spanwise length

Fig. 16 Thickness, spanwise diameter, and liquid column center of mass as functions of nondimensional time for various $W e$ conditions.

\section{Conclusions}

We simulated liquid column deformation for high We behind a shockwave.

Relatively large oscillations of the drag coefficient were observed for $W e=500$ and 1000 , which were the relatively low-We conditions used in this study. The Strouhal number obtained from the present simulation was close to that of a rigid column. Hence, the drag coefficient was assumed to oscillate because of the pressure of the liquid interface; it was periodically varied due to vortices at the wake as well as the rigid column.

The liquid column became flattened in high-speed gas flow. Liquid column flattening may be encouraged by pressure fluctuations at the downstream interface induced by the wake for $W e=1000$. This suggests that the pressure fluctuation at the downstream interface owing to the wake affects the liquid column deformation and the drag coefficient.

In the future, a three-dimensional simulations will be conducted to quantify the drag coefficient and to deform the drop shape for large-scale simulation.

\section{References}

Aulisa, E., Manservisi, S. and Scardovelli, R., A novel representation of the surface tension force for two-phase flow with reduced spurious currents, Computer methods in applied mechanics and engineering, Vol.195 (2006), pp.6239-6257.

Blevins, R.D., Flow-induced vibration. $2^{\text {nd }}$ ed, New York, (1990).

Bo, W., Liu, X., Glimm, J. and Li, X., A robust front tracking method: verification and application to simulation of the primary breakup of a liquid jet, SIAM Journal on Scientific Computing, Vol.33, No.4 (2011), pp.1505-1524.

Broumand, B. and Birouk, M., Liquid jet in a subsonic gaseous crossflow: recent progress and remaining challenges, Progress in Energy and Combustion Science, Vol.57 (2016), pp.1-29.

Chandrasekhar, S., Hydrodynamic and hydromagnetic stability (1981), Dover..

Chang, C.H., Deng, X. and Theofanous, T.G., Direct numerical simulation of interfacial instabilities: A consistent, conservative, all-speed, sharp-interface method. Journal of Computational Physics, Vol.242 (2013), pp.457-492.

Chen, H., Two-dimensional simulation of stripping breakup of a water droplet. AIAA Journal, Vol.46 (2008), pp.11351143.

Fedkiw, R.P., Aslam, T., Merriman, B. and Osher, S., A non-oscillatory Eulerian approach to interfaces in multimaterial flows (the ghost fluid method), Journal of Computational Physics, Vol.152, No.2 (1999), pp.457-492.

Flock, A.K., Guildenbecher, D.R., Chen, J., Sojika, P.E. and Bauser, H.-J., Experimental statistics of droplet trajectory and air flow during aerodynamic fragmentation of liquid drops, International Journal of Multiphase Flow, Vol.47 (2012), pp. 37-49.

Han, J. and Tryggvason, G., Secondary breakup of axisymmetric liquid drops. I. Acceleration by a constant body force, Physics of Fluids, Vol.11, No.12 (1999), pp.3650-3667.

Han, J. and Tryggvason, G., Secondary breakup of axisymmetric liquid drops. II. Impulsive acceleration, Physics of 
Fluids, Vol.13, No.6 (2001), pp.1554-1565.

Hsiang, L.P. and Faeth, G.M., Drop deformation and breakup due to shock wave and steady disturbances, International Journal of Multiphase Flow, Vol.21, No.4 (1995), pp.545-560.

Igra, D. and Takayama, K., Numerical simulation of shock wave interaction with a water column, Shock Waves, Vol.11, No.3 (2001), pp.219-228.

Imamura, O., Ishikawa, Y., Suzuki, S., Hukumoto, K., Nishida, S., Ujiie, Y. and Tsue, M., Combustion characteristics of liquid normal alkane fuels in a model combustor of supersonic combustion ramjet engine, Transactions of Japan Society for Aeronautical and Space Sciences, Vol.58, No.675 (2010), pp.116-122 (in Japanese).

Inamura, T., Yanaoka, H. and Kawada, T., Visualization of airflow around a single droplet deformed in an airstream, Atomization and Sprays, Vol.19, No.7 (2009), pp.667-677.

Joseph, D.D., Belanger, J. and Beavers, G.S., Breakup of a liquid drop suddenly exposed to high-speed airstream. International Journal of Multiphase Flow, Vol.25 (1999), pp.1263-1303.

Kamiya, T., Asahara, M., Hattori, Y., Miyasaka, T. and Asato, K., Evaluation of breakup initiation time by visualized image of liquid drop breakup in high velocity gas flow, Atomization: journal of the ILASS-Japan, Vol. 28, No.95 (2019), pp.78-85 (in Japanese).

Liu, N., Wang, Z., Sun, M., Wang, H. and Wang, B., Numerical simulation of liquid droplet breakup in supersonic flows, Acta Astronautica, Vol.145 (2018), pp.116-130.

Meng, J.C. and Colonius, T., Numerical simulations of the early stages of high-speed droplet breakup, Shock Waves, Vol.25, No.4 (2015), pp.399-414.

Meng, J.C. and Colonius, T., Numerical simulation of the aerobreakup of a water droplet, Journal of Fluid Mechanics, Vol.835 (2018), pp.1108-1135.

Osaka, J., Uriuda, Y., Imamura, O., Yamashita, K., Takahashi, S., Tsue, M. and Kono, M., Combustion characteristics of kerosene in a scramjet combustor, Transactions of Japan Society for Aeronautical and Space Sciences, Vol.55, No.637 (2007), pp.98-103 (in Japanese).

Park, S.W., Kim, S. and Lee C.S., Breakup and atomization characteristics of mono-dispersed diesel droplets in a crossflow air stream, International Journal of Multiphase Flow, Vol.32 (2006), 807-822.

Quan, S. and Schmidt, D.P, Direct numerical study of a liquid droplet impulsively accelerated by gaseous flow, Physics of Fluids, Vol.18, 102103 (2006).

Ranger, A.A. and Nicholls, J.A.: Aerodynamic shattering of liquid drops. In: Proceeding AIAA 6th Aerospace Sciences Meeting, (1968), pp.68-83.

Sembian, S., Liverts, M., Tillmark, N. and Apazidis, N., Plane shock wave interaction with a cylindrical water column. Physics of Fluids, Vol.28, 056102 (2016).

Shao, C., Luo, K. and Fan, J., Detailed numerical simulation of unsteady drag coefficient of deformable droplet, Chemical Engineering Journal, Vol.308 (2017), pp.619-631.

Shyue, K.M., A wave-propagation based volume tracking method for compressible multicomponent flow in two space dimensions, Journal of Computational Physics, Vol.215, No.1 (2006), 219-244.

Sussman, M., Smereka, P., and Osher, S., A level set approach for computing solutions to incompressible two-phase flow, Journal of Computational Physics, Vol.114, No.1 (1994), pp.146-159.

Terashima, H. and Tryggvason, G., A front-tracking method with projected interface conditions for compressible multifluid flows, Computers \& Fluids, Vol.39, No.10 (2010), pp.1804-1814.

Theofanous, T.G., Li, G.J. and Dinh, T.N., Aerobreakup in rarefied supersonic gas flow, Trans, ASME Journal of Fluids Engineering, Vol.126, No.4 (2004), pp.516-527.

Theofanous, T. G. and Li, G. J., On the physics of aerobreakup, Physics of Fluids, Vol.20, 052103 (2008).

Theofanous, T.G., Mitkin, V.V., Ng, C.L., Chang, C-H, Deng, X. and Sushchikh, S., The physics of aerobreakup. II. Viscous liquids, Physics of Fluids, Vol.24, 022104 (2012).

Toro, E. F., Spruce, M. and Speares, W., Restoration of the contact surface in the HLLC-Riemann solver, Shock Waves, Vol. 4, No.1 (1994), pp.25-34.

Van Leer, B., Towards the ultimate conservation difference scheme. IV. A new approach to numerical convection, Journal of Computational Physics, Vol.23, No.3, (1977), pp.276-299.

Wadhwa, A.R., Magi, V. and Abraham, J., Transient deformation and drag of decelerating drops in axisymmetric flow. Physics of Fluids, Vol.19, 113301 (2007). 